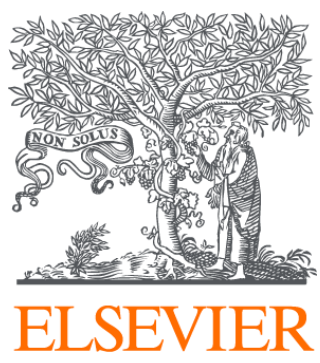

Since January 2020 Elsevier has created a COVID-19 resource centre with free information in English and Mandarin on the novel coronavirus COVID-

19. The COVID-19 resource centre is hosted on Elsevier Connect, the company's public news and information website.

Elsevier hereby grants permission to make all its COVID-19-related research that is available on the COVID-19 resource centre - including this research content - immediately available in PubMed Central and other publicly funded repositories, such as the WHO COVID database with rights for unrestricted research re-use and analyses in any form or by any means with acknowledgement of the original source. These permissions are granted for free by Elsevier for as long as the COVID-19 resource centre remains active. 
Results: Expert feedback was incorporated into the tool, with consensus achieved after four survey rounds. Three categories of HA-SAB, with tailored organisational responses for each classification, were established in the tool. 'Highly preventable' infections involved a clear breach in IP practices, and 'possibly preventable' infections were associated with healthcare interventions where a specific IP breach was not evident. 'Not actionable' events were those where the source of infection does not appear amenable to local change. Pilot application of the completed tool found that $56 \%$ of HA-SAB events were highly or possibly preventable, with modifiable factors not identified in the remainder of reviewed cases.

Conclusion: A Prevention Assessment and Response Tool was successfully developed using a Delphi Technique to assist organisations with investigating and responding to HA-SAB events, and to identify future priority areas for $\mathrm{HA}-\mathrm{SAB}$ reductions. Wider use of the tool is required to evaluate utility and impact on patient outcomes.

14

\section{STRENGTHENING BIO-PREPAREDNESS FOR MANAGING PATIENTS WITH SUSPECTED HIGH CONSEQUENCE INFECTIOUS DISEASE (HCID) USING SIMULATION AND VIDEO-REFLEXIVE METHODS}

Mary Wyer ${ }^{1,2,3}$, Su-Yin Hor ${ }^{4}$, Ruth Barratt ${ }^{1}$, Patricia Ferguson ${ }^{2,6}$, Ramon Shaban $^{2,3,5,6}$, Kavita Varshney ${ }^{2,7}$, Lyn Gilbert ${ }^{3,7}$

${ }^{1}$ Westmead Institute For Medical Research, Westmead, Australia

${ }^{2}$ New South Wales Biocontainment Centre, Western Sydney Local Health

District and New South Wales Health, Westmead, Australia

${ }^{3}$ Marie Bashir Institute for Infectious Diseases and Biosecurity, University of Sydney, Sydney, Australia

${ }^{4}$ University of Technology, Sydney, Australia

${ }^{5}$ Faculty of Medicine and Health, Susan Wakil School of Nursing and Midwifery, University of Sydney, Camperdown, Australia

${ }^{6}$ Division of Infectious Diseases and Sexual Health, Westmead Hospital and Western Sydney Local Health District, Westmead, Australia

${ }^{7}$ University of Sydney, Sydney, Australia

Introduction: The NSW Biocontainment Centre (NBC) at Westmead will soon become the state-wide facility for the care of patients with high consequence infectious diseases (HCID). Researchers have collaborated with NBC staff and other stakeholders to adapt current local health district HCID procedures, for the care of patients with viral haemorrhagic disease, for the new NBC.

Methods: Several HCID exercises were designed on paper, by NBC clinicians, drawing on local policy as well as international biocontainment centre procedures. Exercises focused on procedures within quarantine rooms including: mobile $\mathrm{x}$-rays; preparation of blood samples for transfer to a PC4 laboratory; removal of a deceased person; and healthcare worker collapse. Simulations of these exercises were video-recorded between February and June 2021. After each exercise, the clinicians involved, and colleagues, collaboratively reviewed and analysed the recordings, in researcher-facilitated reflexive discussions. The aim was to identify how optimal infection prevention and biocontainment could be maintained while performing complex procedures. Suggestions for practice optimisation were then tested in subsequent video-recorded simulations and repeated until consensus on optimal practice was achieved.

Results: Preliminary analysis of reflexive discussions shows that the combination of simulation and video-reflexivity enabled participants to identify infection and occupational risks through collaborative analysis of exercise footage. Video-reflexive methods also enabled participants to recognise, discuss and test alternative viewpoints on what constitutes safe and effective practices, leading to adjustments where appropriate, and consensus on adapted guidelines.

Conclusion: Video-reflexivity enables context-sensitive and consensus-building co-design of policies and procedures, critical to the preparation for a new biocontainment unit.

\section{7}

USE OF PORTABLE AIR CLEANERS TO REDUCE AEROSOL TRANSMISSION ON A HOSPITAL COVID-19 WARD

Caroline Marshall ${ }^{1,2,3}$, Kirsty Buising ${ }^{1,2,3}$, Robyn Schofield ${ }^{2}$, Louis Irving ${ }^{1}$, Melita Keywood ${ }^{4}$, Ashley Stevens ${ }^{1}$, Nick Keogh ${ }^{1}$,
Grant Skidmore ${ }^{2}$, Imogen Wadlow ${ }^{2}$, Kevin Kevin ${ }^{2}$

Behzad Rizmanchi ${ }^{2}$, Amanda Wheeler ${ }^{5}$, Ruhi Humphries ${ }^{4}$, Marion Kainer ${ }^{6}$, Forbes McGain ${ }^{6}$, Jason Monty ${ }^{2}$

${ }^{1}$ The Royal Melbourne Hospital, Melbourne, Australia

${ }^{2}$ The University of Melbourne, Melbourne, Australia

${ }^{3}$ Peter Doherty Institute for Infection and Immunity, Melbourne, Australia

${ }^{4}$ Common Wealth Scientific and Industrial Research Organization,

Melbourne, Australia

${ }^{5}$ Australian Catholic University, Melbourne, Australia

${ }^{6}$ Western Health, Melbourne, Australia

Introduction: It is now accepted that COVID-19 can be spread via the airborne route. Hospital ventilation systems are largely designed for comfort and many areas may not be suitable for managing patients with airborne infections. We traced airflow in a COVID-19 ward and tested the effectiveness of commercially available air cleaners in reducing airborne particle concentrations.

Methods: Testing was performed in a ward which had previously housed COVID-19 patients. The return air vent for the whole ward is just inside the single entrance and exit point. Glycerine-based aerosol was used as a surrogate for respiratory aerosols. The transmission of aerosols from a single patient room into corridors and a nurses' station in the ward was measured. The rate of clearance of aerosols was measured over time from the patient room, nurses' station and ward corridors with and without air cleaners (also called portable HEPA filters).

Results: Aerosols rapidly travelled from the patient room into other parts of the ward. Air cleaners were effective in increasing the clearance of aerosols from the air in clinical spaces and reducing their spread to other areas. With two small domestic air cleaners in a single patient room of a hospital ward, $99 \%$ of aerosols could be cleared within 5.5 minutes.

Conclusion: Air cleaners may be useful in clinical spaces to help reduce the risk of healthcare acquired acquisition of respiratory viruses that are transmitted via aerosols. They are easy to deploy and are likely to be cost effective in a variety of healthcare settings.

A HOSPITAL-WIDE RESPONSE TO MULTIPLE OUTBREAKS OF COVID-19 IN HEALTH CARE WORKERS: LESSONS LEARNED FROM THE FIELD

Caroline Marshall $1^{1,2,3}$, Kirsty Buising ${ }^{1,2,3}$, Deborah Williamson ${ }^{1,2,3}$, Benjamin Cowie ${ }^{1,3,4}$, Jennifer MacLachlan ${ }^{3,4}$, Elizabeth Orr ${ }^{1}$, Christopher Maclsaac ${ }^{1,2}$, Eloise Williams ${ }^{1}$, Katherine Bond ${ }^{1}$, Stephen Muhi ${ }^{1,2}$, James McCarthy ${ }^{1,2,3}$, Andrea Maier ${ }^{1}$, Louis Irving $^{1}$, Denise Heinjus ${ }^{1}$, Cate Kelly ${ }^{1}$

${ }^{1}$ The Royal Melbourne Hospital, Grattan St, Australia

${ }^{2}$ The University of Melbourne, Parkville, Australia

${ }^{3}$ Peter Doherty Institute for Infection and Immunity, Melbourne, Australia

${ }^{4}$ Victorian Infectious Diseases Reference Laboratory, Melbourne, Australia

Introduction: The Royal Melbourne Hospital (RMH) experienced a significant number of staff COVID-19 infections during July and August 2020 concurrent with a state-wide surge in cases. We describe the epidemiology of the infections and the interventions associated with outbreak control.

Methods: The RMH is a large tertiary hospital on multiple sites including geriatric and rehabilitation beds at the Royal Park Campus (RPC). The Infection Prevention service managed the outbreaks and exposures, including staff and patient contact tracing, in conjunction with the Victorian Department of Health and Human Services using their COVID-19 guidelines and definitions. A staff well-being service was set up to manage infected and furloughed staff. Data were entered into a REDCap database and analysed using Stata 16.

Results: Between 1 July and 31 August 2020, 262 COVID-19 cases occurred in RMH staff including $179(68.3 \%)$ nurses and 38 (14.5\%) support staff. 107 (40.8\%) were at RPC and coincided with a large number of patient infections (peak of 60). $57(21.8 \%)$ infections occurred on COVID-19 wards, the emergency department and intensive care unit. Twenty $(7.6 \%)$ occurred on "cold" wards which experienced unexpected outbreaks. Mitigation strategies according to a hierarchy of controls were introduced. These included elimination, engineering and administrative controls and personal protective equipment as well as frequent testing of staff and patients with rapid access to results under a previously established governance structure. 